\title{
Seroprevalence of Bartonella in Eastern China and analysis of risk factors
}

\author{
Jimin Sun 1,2, Guiming Fu², Junfen Lin², Xiuping Song ${ }^{1}$, Liang Lu and Qiyong Liu*1
}

\begin{abstract}
Background: Bartonella infections are emerging in the Zhejiang Province of China. However, there has been no effort to date to explore the epidemiology of these infections in this region, nor to identify risk factors associated with exposure to Bartonella. The aim of this study was to investigate the seroprevalence of Bartonella in both patients bitten by dogs and blood donors (for control) in Eastern China, and to identify risk factors associated with exposure to Bartonella. As no previous data for this region have been published, this study will provide baseline data useful for Bartonella infection surveillance, control, and prevention.

Methods: Blood samples were collected from industrial rabies clinic attendees and blood donors living in eight areas of the Zhejiang Province of China, between December 2005 and November 2006. An indirect immunofluorescent antibody test was used to determine the presence of Bartonella in these samples. Risk factors associated with Bartonella exposure were explored using Chi-square tests and logistic regression analysis of epidemiological data relating to the study's participants.

Results: Bartonella antibodies were detected in 19.60\% (109/556) of blood samples. Seroprevalence varied among the eight areas surveys, ranging from over $32 \%$ in Hangzhou to only $2 \%$ in Jiangshan $\left(X^{2}=28.22, P<0.001\right)$. We detected a significantly higher prevalence of Bartonella antibodies in people who had been bitten by dogs than in blood donors $\left(X^{2}=13.86, P<0.001\right)$. Seroprevalence of Bartonella was similar among males $(18.61 \%, n=317)$ and females $(20.92 \%, n$ $=239$ ).

Conclusions: Bartonella antibodies were encountered in people living across Zhejiang Province and the seropositivity rate among those exposed to dog bites was significantly higher than that among blood donors, indicating that dog bites may be a risk factor for Bartonella infection.
\end{abstract}

\section{Background}

The genus Bartonella comprises of fastidious, Gram-negative hemotropic bacteria that infect blood erythrocytes and endothelial cells of their hosts [1]. More than 20 species or subspecies of Bartonella have been described, of which at least 12 are recognized as human pathogens [2]. These species exploit a variety of different mammals as reservoir hosts and arthropods as vectors, and provoke a broad spectrum of manifestations in humans.

According to clinical reports, the number of patients with Bartonella infections in Zhejiang Province is the

* Correspondence: liuqiyong@icdc.cn

1 Department of Vector Biology and Control,State Key Laboratory for Infectious Diseases Prevention and Control, National Institute for Communicable Disease Control and Prevention, Chinese Center for Disease Control and Prevention, Beijing 102206, China

Full list of author information is available at the end of the article second largest in China. However, to date, there have been no efforts to explore the epidemiology of these infections in this region, nor to identify risk factors associated with exposure to Bartonella. Directly detecting Bartonella in humans is problematic, but detection of antibodies as an indication of exposure to the agent has been widely used in previous studies. In this study, we adopted the latter approach for the first time to investigate human exposure to Bartonella in the Zhejiang Province of China.

\section{Methods}

\section{Blood samples}

Patient blood samples $(\mathrm{n}=205$ from rabies clinics; $\mathrm{n}=$ 351 from normal blood donors) were collected from eight locations (Hangzhou, Tiantai, Longyou, Huzhou, Jiang- 
shan, Chun'an, Longyou, and Linhai) of the Zhejiang Province of China from December 2005 to November 2006. The aims of our study were explained to all participants upon enrollment, and their consent was obtained prior to inclusion in this study. All enrolled participants provided information upon inclusion in the study, with regard to their age, gender, place of residence, and whether they had been exposed to dog bites.

Experimental research reported in this study has been performed with the approval of the ethics committee of Zhejiang Provincial Center for Disease Control and Prevention. Human research was carried out in compliance with the Helsinki Declaration.

\section{Serological testing}

A commercially available Bartonella henselae (B. henselae)-based indirect immunofluorescence antibody (IFA) test kit (Euroimmun, Order No. FI219b-1005G) was used to assess the presence of IgG antibodies against $B$. henselae in human samples. After the samples were diluted $1: 100,1: 320,1: 1000,1: 3200$, and 1:10000 in phosphatebuffered saline PBS-Tween buffer (provided in the test kit), the IFA assay was conducted following the manufacturer's protocol. Positive and negative controls (provided in the test kit) were also used. Immunofluorescence was observed using an epifluorescence microscope at magnifications of $40 x$ and $200 \times$. According to the manufacturer's guidelines, a titer of 1:320 was considered indicative of an infection, and IgG antibody titers of 1:1000 or higher were judged as an indicator for acute infection.

\section{Data analysis}

Chi-square tests and logistic regression analysis of Bartonella seroprevalence among sites, gender, age groups, and exposure to dog bites were conducted using the SPPS, version 11.0 statistical package (Chicago, IL, USA). The dependent variable in the logistic regression was assigned as the serological status of patients and the independent variables were site, gender, age, exposure, and site $\times$ exposure. The method of logistic regression used was forward-conditional. The stepwise probability was set to 0.05 for entry and 0.10 for removal. the classification cutoff was 0.5 and the maximum number of iterations was 20. Omnibus tests of model coefficients were also conducted.

\section{Results}

Table 1 summarizes the results of positive antibody reactions in patients at various dilution titers of their blood samples. The proportion of titers of 1:1000 or higher among people bitten by dogs was consistently higher than those among healthy people who had not experienced bites. Furthermore, according to the manufacturer's instructions for data analysis, the final resultant mean of the proportion of acute infection in people exposed to dog bites was higher than that in control group.

Blood samples were collected from 556 people living in eight locations across Zhejiang Province (Table 2). Of these, 317 patients were male and 239 were female. Overall, $19.60 \%$ (109/556) of blood samples were seropositive for Bartonella based on antibody immunofluorescence results. Of note, seroprevalence of Bartonella varied significantly among sites within the Zhejiang Province $\left.\left(2.00-32.38 \% ; \mathrm{X}^{2}=28.220, \mathrm{P}<0.001\right]\right)$, and between those patients bitten by dogs versus those not bitten $(27.80 \%$ and $14.81 \%$, respectively; $\left.\mathrm{X}^{2}=13.856, \mathrm{P}<0.001\right)$. Seroprevalence of Bartonella was found to be similar among males $(18.61 \%, \mathrm{n}=317)$ and females $\left(20.92 \%, \mathrm{n}=239\right.$; $\mathrm{X}^{2}$ $=0.461, \mathrm{P}=0.497>0.05)$. Furthermore, participants were divided into four groups according to age and the seroprevalence in each was determined. In summary, we found that $17.78 \%(5 / 18)$ of those patients less than 15 years in age had Bartonella antibody, 18.27\% (59/323) of patients between 15 and 44 years in age had antibodies, $18.42 \%(28 / 152)$ of patients between 45 and 59 years old had antibodies, and $26.98 \%$ (17/63) of those patients over 59 years in age had antibodies. These results suggest that age was an insignificant factor in Bartonella antibody expression $\left(\mathrm{X}^{2}=6.364, \mathrm{P}>0.05\right)$.

The Chi-square value in omnibus tests of model coefficients was determined to be $60.326(\mathrm{P}<0.05)$. Furthermore, the overall correct percentage was found to be $81.5 \%$. Variables in the equation included site, exposure, and sitexexposure. The wald of sitexexposure, site, and exposure were determined to be $25.477(\mathrm{P}=0.001)$, $20.090(\mathrm{P}=0.005)$, and 16.174 $(\mathrm{P}=0.024)$, respectively.

\section{Discussion}

Various Bartonella species, including B. henselae, have previously been detected in domestic cats [3], small mammals, and Haemaphysalis longicornis and Ixodes sinensis ticks [4] in the Zhejiang Province of China. Of note, transmisson of Bartonella-related infection from any of these sources to humans is feasible. Therefore, it is necessary to study the prevalence of Bartonella in people throughout the Zhejiang Province and to analyze relevant risk factors of infection. In our study, the seroprevalence of Bartonella was found to be $14.81 \%$ among healthy patients, a percentage similar to the recently reported percentage (14.28\%) recorded in the Yunnan Province in Southwestern China [5], but much lower than the percentage reported in Beijing (34.5\%) [6]. Furthermore, Kikuchi et al. [7] previously reported a $B$. henselae specific IgG seroprevalence of $3.1 \%$ among adult patients with cardiovascular disease and $10.9 \%$ in a high-risk population of healthy veterinary students in Japan, of whom only $0.8 \%$ had positive results of serologic testing for $B$. 


\begin{tabular}{lccccc}
\hline & & \multicolumn{3}{c}{ Dilution titers } \\
\cline { 2 - 6 } & $\mathbf{1 : 3 2 0}$ & $\mathbf{1 : 1 0 0 0}$ & $\mathbf{1 : 3 2 0 0}$ & $\mathbf{1 : 1 0 0 0 0}$ & Total \\
\hline Bitten by dogs $(\mathrm{n})$ & 30 & 18 & 6 & 3 & 57 \\
Control group $(\mathrm{n})$ & 43 & 8 & 1 & 0 & 52 \\
\hline Total $(\mathrm{n})$ & 73 & 26 & 7 & 3 & 109 \\
\hline
\end{tabular}

henselae specific IgM. The bases for these differences are not clear, but are likely to be multi-factorial.

Due to the inherent serological cross-reactivity among Bartonella species in IFA assays [8,9], the observed seropositivity to $B$. henselae antigen may actually represent a previous or current infection with another Bartonella species. According to our analysis (carried out according to the manufacturer's instruction), cross reactivity with other Bartonella species cannot be ruled out; however, this kit has more than $84 \%$ specificity and $88 \%$ sensitivity for $B$. henselae IgG. As a result, our data suggest that detected antibodies were against all Bartonella species, while the majority of them were against $B$. henselae.

The fact that patients with Bartonella antibodies were detected across all eight study sites of the Zhejiang Province indicates that the general population in this region is at risk of exposure to Bartonella. Of note, the prevalence varied between sites within the province, although Hangzhou, Tiantai, and Longyou had the highest prevalence of positive reactions. Furthermore, the exposure rate due to dog bites, which was identified as a significant determinant of seroprevalence, also varied between sites, and was significantly higher in Hangzhou than other sites, and significantly lower in Jiangshan than other sites. Moreover, sitexexposure fit the equation in logistic regression analysis, indicating that site and exposure results correlated to each other.

We also found that seroprevalence of Bartonella in individuals bitten by dogs was significantly higher than that among unbitten blood donors. However, the explanation of this finding is not altogether obvious, as domestic cats, rather than dogs, are considered a primary reservoir host for Bartonella. It is established that dogs serve as hosts for Ctenocephalides felis, the suspected vector of Bartonella. Therefore, dogs may acquire and maintain infected and infectious fleas, thereby, indirectly acting as a source of infection. Alternatively, people living in proximity to dogs may also be more likely to live in proximity to cats. However, it remains a possibility that dog bites themselves may transmit Bartonella infection, as Bartonella DNA has been detected in dog saliva [10].

Previously published studies have indicated that $B$. henselae infections are more common in children than adults. However, we did not see a significantly higher seropositivity among children than adults in our studies. In contrast, in our study the highest seropositivity was observed in the oldest ( $>59$ years) patient age group. Others have reported similar findings [11].

Table 2: Zhejiang Province site locations and seroprevalence incidence.

\begin{tabular}{|c|c|c|c|c|c|c|c|}
\hline Site & Latitude & Longitude & Male (n) & Female (n) & Total (n) & $\begin{array}{l}\text { Bartonella } \\
\text { Positive (n) }\end{array}$ & $\begin{array}{l}\text { Positive } \\
\text { rate (\%) }\end{array}$ \\
\hline Hangzhou & $30.15^{\circ} \mathrm{N}$ & $120.20^{\circ} \mathrm{E}$ & 59 & 46 & 105 & 34 & 32.38 \\
\hline Tiantai & $29.14^{\circ} \mathrm{N}$ & $121.03^{\circ} \mathrm{E}$ & 51 & 53 & 104 & 27 & 25.96 \\
\hline Longyou & $29.04^{\circ} \mathrm{N}$ & 119.19E & 30 & 20 & 50 & 11 & 22.00 \\
\hline Huzhou & $20.52^{\circ} \mathrm{N}$ & $120.04^{\circ} \mathrm{E}$ & 34 & 18 & 52 & 8 & 15.38 \\
\hline Jiangshan & $28.73^{\circ} \mathrm{N}$ & $118.61^{\circ} \mathrm{E}$ & 30 & 20 & 50 & 1 & 2.00 \\
\hline Chun'an & $29.61^{\circ} \mathrm{N}$ & $118.84^{\circ} \mathrm{E}$ & 47 & 40 & 87 & 14 & 16.09 \\
\hline Longquan & $28.09^{\circ} \mathrm{N}$ & $119.12^{\circ} \mathrm{E}$ & 31 & 21 & 52 & 8 & 15.38 \\
\hline Linhai & $28.86^{\circ} \mathrm{N}$ & $121.12^{\circ} \mathrm{E}$ & 35 & 21 & 56 & 6 & 10.71 \\
\hline & Total & & 317 & 239 & 556 & 109 & 19.60 \\
\hline
\end{tabular}




\section{Conclusions}

Bartonella infections can be difficult to diagnose clinically, particularly as many of the clinical presentations associated with them have nonspecific symptoms, such as fever. Thus, reliance on clinical reports from physicians to determine the public health burden of infection is not feasible. In contrast, serological surveys of individuals offer a means of quantifying the level of exposure to Bartonella among the population. In this study, we used this approach to demonstrate that exposure to this pathogen is both common and widespread across the Zhejiang Province of China, although prevalence was higher in some parts of the province than others. Furthermore, it appeared that people bitten by dogs were significantly more likely to have Bartonella antibodies than blood donors without exposure to bites. This information should serve to help those in relevant settings to consider Bartonella infection in the differential diagnosis of their patients.

\section{Competing interests}

The authors declare that they have no competing interests.

\section{Authors' contributions}

IS designed the study and drafted the manuscript. GF carried out the patient blood sample collection. JL performed analysis and interpretation of data. XS carried out serological testing. LL participated in the design of the study and helped draft the manuscript. QL conceived of the study and participated in its design and coordination. All authors read and approved the final manuscript.

\section{Acknowledgements}

We thank Guojing Si, Fengxia Meng, Haixia Wu, Dongmei Li, Dongsheng Ren, Jun Wang, Baoxin Cui, and Xiaoran Yang for their help. We also extend appreciation to the Hangzhou Center for Disease Control and Prevention (CDC), Wenzhou CDC, Tiantai CDC, Jindong CDC, Longquan CDC, Linhai CDC, Chun'an CDC, Shangyu CDC, Longyou CDC, Ouhai CDC, Jiande CDC and Jiangshan CDC for their help.

This study was supported by the National Natural Science Foundation of China (NSFC) grant 30371246 and the Project of the State Scientific \& Technological Development of the 10th Five Year Plan grant 2003BA712A09-02.

\section{Author Details}

'Department of Vector Biology and Control,State Key Laboratory for Infectious Diseases Prevention and Control, National Institute for Communicable Disease Control and Prevention, Chinese Center for Disease Control and Prevention, Beijing 102206, China and 2Zhejiang Provincial Center for Disease Control and Prevention, Hangzhou 310051, China

Received: 13 November 2009 Accepted: 20 May 2010

Published: 20 May 2010

\section{References}

1. Boulouis HJ, Chang CC, Henn JB, Kasten RW, Chomel BB: Factors associated with the rapid emergence of zoonotic Bartonella infections. Vet Res 2005, 36:383-410.

2. Henn JB, Gabriel MW, Kasten RW, Brown RN, Theis JH, Foley JE, Chomel BB: Gray foxes (Urocyon cinereoargenteus) as a potential reservoir of a Bartonella clarridgeiae-like bacterium and domestic dogs as part of a sentinel system for surveillance of zoonotic arthropod-borne pathogens in northern California. J Clin Microbio/ 2007, 45:2411-2418.

3. Guiming Fu, Jimin Sun, Qiyong Liu, Tianci Yang, Zhangyao Ren, Gangqiang Ding: Epidemiology investigation of Bartonella henselae in cats from Zhejiang Province. Chinese Journal of Vector Biology and Control 2008, 19:138-140.
4. Sun J, Liu Q, Lu L, Ding G, Guo J, Fu G: Coinfection with four genera of bacteria (Borrelia, Bartonella, Anaplasma, and Ehrlichia) in Haemaphysalis longicornis and Ixodes sinensis ticks from China. Vector Borne Zoonotic Dis 2008, 8:791-796.

5. Hui Yang, Heming B, Falian Y, Binbin Y: Serological survey on Bartonella infection in Yunnan. Chinese Journal of Natural Medicine 2007, 9:277-280.

6. Yang XR, Liu QY, Cui BY, Wang LX, Peng ZH, Ren DS: Using direct enzyme linked immunosorbent assay for the detection of $\lg G$ antibody on Bartonella henselae among healthy people in Changping, Beijing. Zhonghua Liu Xing Bing Xue Za Zhi 2007, 28:688-691.

7. Kikuchi E, Maruyama S, Sakai T, Tanaka S, Yamaguchi F, Hagiwara T: Serological investigation of Bartonella henselae infection in clinically cat scratch disease-suspected patients, patients with cardiovascular diseases and healthy veterinary students in Japan. Microbiol Immunol 2002, 46:313-316.

8. Dalton MJ, Robinson LE, Cooper J, Regnery RL, Olson JG, Childs JE: Use of Bartonella antigens for serologic diagnosis of cat-scratch disease at a national referral center. Arch Intern Med 1995, 155:1670-1676.

9. La Scola B, Raoult D: Serological cross-reactions between Bartonella quintana, Bartonella henselae, and Coxiella burnetii. J Clin Microbiol 1996, 34:2270-2274.

10. Duncan AW, Maggi RG, Breitschwerdt EB: Bartonella DNA in dog saliva. Emerg Infect Dis 2007, 13:1948-1950.

11. Pandak N, Dakovic-Rode, Cabraja I, Kristof Z, Kotarac S: Prevalence of Bartonella henselae antibodies in children and blood donors in Croatia. Infection 2009, 37:166-167.

Pre-publication history

The pre-publication history for this paper can be accessed here: http://www.biomedcentral.com/1471-2334/10/121/prepub

doi: 10.1186/1471-2334-10-121

Cite this article as: Sun et al., Seroprevalence of Bartonella in Eastern China and analysis of risk factors BMC Infectious Diseases 2010, 10:121

\section{Submit your next manuscript to BioMed Central} and take full advantage of:

- Convenient online submission

- Thorough peer review

- No space constraints or color figure charges

- Immediate publication on acceptance

- Inclusion in PubMed, CAS, Scopus and Google Scholar

- Research which is freely available for redistribution
C Biomed Central 